\title{
Transcriptomic Effects of Tet-On and Mifepristone-Inducible Systems in Mouse Liver
}

\author{
Mercedes Reboredo, ${ }^{1,2}$ Maria Gabriela Kramer, ${ }^{1,3}$ Cristian Smerdou, ${ }^{1}$ \\ Jesús Prieto, ${ }^{1,2}$ and Javier De Las Rivas ${ }^{4}$
}

\begin{abstract}
Control of transgene expression from long-term expression vectors can be achieved with inducible and regulated promoters. The two most commonly used inducible systems employ doxycycline or mifepristone as the drug activating a silent trans-activator, which is expressed from a constitutive promoter. We evaluated the alterations provoked by constitutive expression in the liver of rtTA2 ${ }^{\mathrm{S}}-\mathrm{M} 2$ (rtTA2; second-generation reverse tetracycline-controlled trans-activator) and GLp65, which are the trans-activators of the doxycyline- and mifepristone-inducible systems, respectively. To this end we performed transcriptomic analysis of mice expressing these trans-activators in the liver over 1 month. rtTA2 expression induced alterations in a few genes (69 gene probesets; false discovery rate [FDR], 0.05), whereas GLp65 caused more numerous changes (1059 gene probe-sets, an FDR of 0.05). However, only 20 and 53 of the genes from the rtTA2 and GLp65 groups, respectively, showed changes ( $R$-fold $\geq 3$ ). Functional assignments indicate that alterations were mild and of little general significance. Few additional transcriptomic changes were observed when expressing trans-activators in the presence of inducer drugs; most were due to the drugs themselves. These results and the absence of toxicity observed in treated animals indicate that the two inducible systems are well tolerated and have little impact on the liver transcriptome profile. The milder alterations found with the use of rtTA2 suggest that this system is possibly safer for gene therapy applications.
\end{abstract}

\section{Introduction}

G ENE THERAPY has emerged as a potential alternative strategy for treating a wide variety of diseases. For many applications long-term expression vectors are desirable. These types of vectors, however, would require the use of inducible and regulable promoters to adapt transgene expression to specific therapeutic needs. Moreover, these types of promoter are essential when the transgene is a molecule with strong biological activity and potential toxicity, as is the case with immunostimulatory cytokines such as interleukin (IL)-12 and interferon (IFN)- $\alpha$ (Melero et al., 2001; Prieto et al., 2004). Among the existing inducible systems, the Tet-regulatory system (Tet-on) and the progesterone receptor regulatory system (RU486-on) have been used most frequently (Harvey and Caskey, 1998). Both methods have been successfully employed in preclinical gene therapy applications to treat liver cancer by expressing IL-12 in liver for a prolonged period of time (Wang et al., 2004; Zabala et al., 2004).

Inducible systems are based on the use of two expression cassettes: one encoding a trans-activator (TA) protein and a second containing the therapeutic gene driven by an inducible promoter that can be activated by the TA. The TA is a chimeric protein that comprises a ligand-binding domain (LBD), a DNA-binding domain (DBD), and a transcriptional activation domain (AD). The TA is expressed constitutively as an inactive form that becomes active on binding of the inducer to the LBD. This enables the TA to bind the inducible promoter through the $\mathrm{DBD}$, thus promoting transcription of the therapeutic transgene by the AD.

The optimal version of the Tet-on system is based on the use of protein $\mathrm{rtTA}^{\mathrm{S}}-\mathrm{M} 2$ (rtTA2; second-generation reverse tetracycline-controlled trans-activator) as TA. rtTA2 contains a tetracycline resistance $\left(\right.$ Tet $\left.^{\mathrm{R}}\right)$ gene carrying five specific mu-

\footnotetext{
${ }^{1}$ Division of Gene Therapy, Center for Applied Medical Research (CIMA) and University Clinic-University of Navarra, 31008 Pamplona, Spain.

${ }^{2}$ Centro de Investigación Biomédica en Red de Enfermedades Hepáticas y Digestivas (CIBERehd), University Clinic, 31008 Pamplona, Spain.

${ }^{3}$ Present address: Peter MacCallum Cancer Research Institute, Cancer Immunology Program, East Melbourne 3001, Australia.

${ }^{4}$ Bioinformatics and Functional Genomics Research Group, Cancer Research Center (IBMCC-CIC), CSIC and University of Salamanca (CSIC/USAL), E37007 Salamanca, Spain.
} 
tations, in which codon usage was optimized for mammalian cells, and from which all potential splice donor and acceptor sites, as well as predicted mRNA hairpin structures, were eliminated (Urlinger et al., 2000). The AD in rtTA2 is formed by three copies of a minimal VP16 12-amino acid domain replacing the original larger VP16 130-amino acid AD present in rtTA (Baron et al., 1997). All these modifications decreased the toxicity and immunogenicity of the TA and reduced the binding of the TA to the inducible promoter in the absence of the inducer drug (Baron et al., 1997). Furthermore, rtTA2 can be fully induced with a 10-fold lower concentration of doxycycline (Dox) as compared with rtTA, limiting potential drug-derived toxicity (Urlinger et al., 2000).

The optimized RU486-on system uses the progesterone antagonist mifepristone (RU-486) as inducer and a chimeric protein named GLp65 as TA (Wang et al., 1994). GLp65 is a fusion of the yeast GAL4 DBD, a human progesterone receptor LBD containing a 19-amino acid carboxy-terminal deletion (PRLBDA), and the 269-amino acid carboxy-terminal acidic AD from human p65 (Burcin et al., 1999). This latter protein is a member of the Rel protein family, usually found as part of NF- $\kappa$ B transcription complexes (Siebenlist et al., 1994).

An important safety requirement for inducible systems is that the TAs, in their active or inactive forms, do not interfere with endogenous gene expression, or with cellular functions. The fact that these chimeric proteins are formed by functional domains derived from potent transcriptional factors could entail the risk of unwanted side effects due to activation of endogenous promoters. In the case of rtTA it is known that the VP16 AD can interact with many essential transcription factors such as human ADA2 coactivator (Candau et al., 1996), TFIIB, TFIIH, TFII40, TBP, and SAGA histone-acetylating complex (Blau et al., 1996; Hall and Struhl, 2002). The reduction of this AD in rtTA2 probably eliminates many potential interactions with cellular transcription factors, but further studies are necessary to analyze its toxicity in vivo. Regarding GLp65, it has been described that its AD can interact with transcription regulation and signaling factors such as TFIIB and TBP (Schmitz et al., 2004), SMRT (Espinosa et al., 2003), CBP (Parry and Mackman, 1997), calmodulin (Antonsson et al., 2003), protein phosphatase-2A (Yang et al., 2001), and PARP-1 and C/EBP $\beta$ (Xia et al., 1997). In addition, the human progesterone receptor LBD present in GLp65 can bind heat shock proteins and transcriptional coactivators (Leonhardt and Edwards, 2002). All these potential interactions could contribute to the toxicity of TAs, especially taking into account that they will be expressed constitutively as long as the vector remains in the transduced tissue.

Although no signs of toxicity derived from TA expression have been observed in studies performed in vivo with the optimized Tet-on or RU486-on inducible system (Wang et al., 2004; Zabala et al., 2004), the effects that prolonged expression of their respective TAs could have in the transduced organ have not been evaluated in detail. Because of the potential therapeutic applications of both inducible systems we performed genome-wide microarray analysis in mice that had expressed the respective TAs in the liver for 1 month. This study showed only moderate changes in the gene expression profile of liver cells in the presence of rtTA2. Transcriptomic changes were more numerous in the case of GLp65 but no morphological or biochemical changes were observed in the liver with either of the two TAs. Because transcription regulation responds to mechanisms that are highly conserved among mammals we believe that our data could anticipate similar effects in humans.

\section{Materials and Methods}

\section{Animal manipulation}

Five- to 6-week-old female BALB/c mice were purchased from Harlan Laboratories (Barcelona, Spain). Animals were maintained under standard conditions and all procedures were approved by the institutional ethics committee of the University of Navarra (Pamplona, Spain). The hydrodynamics (HD)-based procedure (Zhang et al., 1999) was used to inject mice with $75 \mu \mathrm{g}$ of plasmid DNA resuspended in 2 $\mathrm{ml}$ of saline $(0.9 \% \mathrm{NaCl})$. Animals receiving the inducer drugs were injected intraperitoneally with doxycycline (Dox; Sigma-Aldrich, St. Louis, MO) at a dose of $50 \mu \mathrm{g} / \mathrm{g}$ in 200 $\mu \mathrm{l}$ of saline solution or with mifepristone (RU-486; SigmaAldrich) at a dose of $250 \mu \mathrm{g} / \mathrm{kg}$ in $60 \mu \mathrm{l}$ of sesame oil. Inducers were administered on a daily basis during the 3 days before sacrifice. Blood samples were collected at the indicated time points by retro-orbital bleeding under inhalatory anesthesia (isoflurane [Forane]; Abbott Laboratories, Abbott Park, IL). Serum was recovered by double centrifugation at $10,000 \mathrm{rpm}$ for $5 \mathrm{~min}$ and stored at $-20^{\circ} \mathrm{C}$ until protein measurement.

\section{Plasmid construction and purification}

EA-rtTA2 and EA-GLp65 plasmids derive from a pGL3basic vector in which the trans-activator (TA) genes rTA2 ${ }^{\mathrm{S}}$ M2 and GLp65, respectively, have been cloned downstream of the EalbPa1AT liver-specific promoter (Kramer et al., 2003) (Fig. 1A). EA-rtTA2 was generated as described previously (Zabala et al., 2004). EA-rtTA2 carries a mutated improved version of rtTA (rtTA2 $\left.{ }^{\mathrm{S}}-\mathrm{M} 2\right)$ (Urlinger et al., 2000). In addition, EA-rtTA2 contains a shortened version of the VP16 domain with lower cell toxicity. EA-GLp65 was generated by inserting the GLp65 gene from pSwitch plasmid (Invitrogen, Carlsbad, CA) into the XbaI and HindIII sites in EalbPa1AT-luc (Kramer et al., 2003). GLp65 is a chimeric protein that contains three functional domains: the region for binding to GAL4 sites in the inducible promoter, a mutated human progesterone receptor for binding to RU-486, and the p65 TA from the NF- $\kappa$ B transcription factor. P ${ }_{\text {GFPwpre }}$ plasmid contains the green fluorescent protein (GFP)-encoding gene linked to the woodchuck hepatitis posttranscriptional regulatory element (WPRE) downstream of the cytomegalovirus (CMV) promoter. The expression cassette CMVGFP-wpre was isolated from pRRLsin18ppt with HindIII and EcoRI and cloned into pGL3basic digested with the same enzymes to generate the $\mathrm{P}_{\mathrm{GFP}}$ wpre plasmid. $\mathrm{pCMV} \beta$ was purchased from Clontech (Mountain View, CA). In all cases plasmid DNA was purified from bacteria culture with an EndoFree plasmid maxi kit (Qiagen, Hilden, Germany) and stored in Tris-EDTA (TE) buffer solution at $-20^{\circ} \mathrm{C}$.

\section{RNA isolation and RT}

Animals were killed and liver samples were collected, rapidly frozen in liquid nitrogen, and kept at $-80^{\circ} \mathrm{C}$. For RNA extraction, a portion of liver tissue was homogenized 
in $1 \mathrm{ml}$ of TRIzol reagent (Invitrogen) with an Ultra-Turrax driver T.25 (Janke \& Kunkel/Ika-Labortechnik, Staufen, Germany), processed according to the manufacturer's protocol, and stored at $-80^{\circ} \mathrm{C}$ until analysis. Samples for microarray analysis were also purified with an RNeasy mini kit (Qiagen). Genomic DNA was eliminated from the samples by treatment with DNase I (Invitrogen). For reverse transcriptase polymerase chain reactions (RT-PCRs) cDNA was synthesized with $2 \mu \mathrm{g}$ of total RNA in 50- $\mu$ l reaction mixtures containing $0.8 \mathrm{mM}$ dNTP mix, $5.3 \mathrm{mM} \mathrm{p}(\mathrm{dN})_{6}$ random primer (Roche, Basel Switzerland), $4 \mathrm{mM}$ dithiothreitol (DTT; Invitrogen), 48 units of RNase inhibitor (Invitrogen), 240 units of Moloney murine leukemia virus (M-MLV) reverse transcriptase (Invitrogen), and $10 \mu \mathrm{l}$ of reaction buffer provided by the enzyme supplier. Reactions were incubated at $37^{\circ} \mathrm{C}$ for $1 \mathrm{hr}$ and at $95^{\circ} \mathrm{C}$ for $1 \mathrm{~min}$.

\section{Quantitative polymerase chain reaction}

cDNA was quantified with iQ SYBR green supermix in an iQ5 real-time PCR detection system (Bio-Rad, Hercules, CA). Primers were designed (Table 1) to discriminate between genomic and cDNA sequences, except those specific for plasmid amplicons. PCR amplification was performed under the following conditions: one cycle of $3 \mathrm{~min}$ at $95^{\circ} \mathrm{C}$; followed by 40 cycles of $15 \mathrm{sec}$ at $95^{\circ} \mathrm{C}, 30 \mathrm{sec}$ at $60^{\circ} \mathrm{C}$ (annealing temperature of $58^{\circ} \mathrm{C}$ when amplifying Vim), $30 \mathrm{sec}$ at $72^{\circ} \mathrm{C}$, and $10 \mathrm{sec}$ at $81^{\circ} \mathrm{C}$ (detection temperature of $79^{\circ} \mathrm{C}$ for GLp65 and Bche); followed by a single final extension cycle of $72^{\circ} \mathrm{C}$ for 4 min. Immediately after the PCR, a melting curve was gen- erated by raising the incubation temperature from 55 to $95^{\circ} \mathrm{C}$ to confirm amplification specificity. The final PCR product was also analyzed by agarose electrophoresis. Samples were analyzed in duplicate and mRNA expression data were normalized by comparison with phosphoglycerate kinase-1 (Pgk1) as an internal control, after checking for the high stability of expression of this gene under all experimental conditions (data not shown). The amount of each transcript was

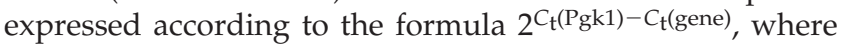
$C_{t}$ is the point at which the fluorescence rises appreciably above background fluorescence.

\section{RNA isolation, cDNA synthesis, and microarray hybridization}

RNA was purified from mouse liver with a commercial kit (Rneasy; Qiagen). RNA concentration was measured at $260 \mathrm{~nm}$ (Ultrospec 2000; Pharmacia Biotech, Uppsala, Sweden) and its purity and quality were determined with an RNA 6000 Nano Chip kit (Agilent Technologies, Palo Alto, CA). RNA was then used to synthesize complementary RNA (cRNA) probes for hybridization to Affymetrix GeneChip mouse genome 430A 2.0 high-density oligonucleotide microarrays (Affymetrix, Santa Clara, CA), which include 22,690 oligonucleotide probe sets corresponding to about 14,000 mouse genes, according to protocols described in the Gene Expression Analysis Technical Manual (see http://www.affymetrix.com/support/techni$\mathrm{cal} / \mathrm{manual} /$ expression_manual.affx [accessed October 2008]). In brief, total RNA was reverse transcribed into doublestranded complementary DNA (cDNA) with an oligo(dT) 24

Table 1. Primers Used in Study

\begin{tabular}{|c|c|c|c|}
\hline Gene symbol & GenBank accession no. & Primer sequence & Fragment length $(b p)$ \\
\hline \multicolumn{4}{|c|}{ mRNA } \\
\hline Mouse Mat1a & NM_133653 & $\begin{array}{l}\text { Sense: 5'-CTCTGGTGTCCTTCCCTGGC-3' } \\
\text { Antisense: 5'-GGTACTTGGCTGGCACCACA-3' }\end{array}$ & 200 \\
\hline Mouse Col1a2 & NM_007743 & $\begin{array}{l}\text { Sense: 5'-TGCTCAGCTTTGTGGATACG-3' } \\
\text { Antisense: 5'-CCAGCAGGTCCTTGGAAACC-3' }\end{array}$ & 370 \\
\hline Mouse Orm2 & MN_011016 & $\begin{array}{l}\text { Sense: 5'-CAATGAGACCCTGAGCTGG-3' } \\
\text { Antisense: 5'-CTTGGAGAGGGTCCCATTCT-3' }\end{array}$ & 230 \\
\hline Mouse Vim & NM_011701 & $\begin{array}{l}\text { Sense: 5'-ACCTGGCCGAGGACATCAT-3' } \\
\text { Antisense: 5'-ATCTGGACATGCTGTTCCTG-3' }\end{array}$ & 238 \\
\hline Mouse Saa3 & NM_011315 & $\begin{array}{l}\text { Sense: 5'-CAGGATGAAGCCTTCCATTG-3' } \\
\text { Antisense: 5'-AGCCTCTCTGGCATCACTGA-3' }\end{array}$ & 249 \\
\hline Mouse Nr3c1 & NM_008173 & $\begin{array}{l}\text { Sense: 5'-GGAGAGGACAACCTGACTTCC-3' } \\
\text { Antisense: 5'-TTGTGCTGTCCTTCCACTGC-3' }\end{array}$ & 248 \\
\hline Mouse Cfh & NM_009888 & $\begin{array}{l}\text { Sense: 5'-CAGGCTCGTGGTCAGAACAA-3' } \\
\text { Antisense: 5'-CCATCTGCACCACATTCACG-3' }\end{array}$ & 298 \\
\hline Mouse Bche & NM_009738 & $\begin{array}{l}\text { Sense: 5'-TGGATGGGAGTGATGCATGG-3' } \\
\text { Antisense: 5'-CACATTGTGCTATTGCCCTGG-3' }\end{array}$ & 173 \\
\hline Mouse Pgk1 & NM_008828 & $\begin{array}{l}\text { Sense: 5'-GGACAAGCTGGACGTGAAGG-3' } \\
\text { Antisense: 5'-ACAGAACATCCTTGCCCAGC-3' }\end{array}$ & 256 \\
\hline \multicolumn{4}{|c|}{ Plasmid } \\
\hline rtTA2S-M2 & & $\begin{array}{l}\text { Sense: 5'-GTCGGTATCGAAGGCCTGACG-3' } \\
\text { Antisense: 5'-ACAGGAACGCGAGCTGATTT-3' }\end{array}$ & 304 \\
\hline GLp65 & & $\begin{array}{l}\text { Sense: 5'-TGAACAGCGGATGAAAGAATCA-3' } \\
\text { Antisense: 5'-TTGACCTCATCTCCTCAAACTG-3' }\end{array}$ & 185 \\
\hline
\end{tabular}


primer containing a T7 polymerase promoter-binding site (Genset Oligos, La Jolla, CA). cDNA was then used as a template to synthesize cRNA by in vitro transcription with T7 MEGAscript (Ambion, Austin, TX), in the presence of biotinylated nucleotides (Enzo Diagnostics, Farmingdale, NY). Labeled cRNAs were fragmented and hybridized to GeneChip mouse genome 430A 2.0, using a Fluidics Station 450 (Affymetrix). Hybridized arrays were stained with streptavidin-phycoerythrin, rewashed, treated with biotinylated antistreptavidin-phycoerythrin antibodies, and restained with streptavidin-phycoerythrin, according to the manufacturer's protocols. The stained arrays were finally scanned in a GeneArray scanner (Hewlett Packard, Palo Alto, CA).

Microarray data analysis: normalization, signal calculation, significant differential expression, and sample/gene profiles clustering

Microarray data analysis was performed according to the following strategy and methods. The RMA (robust multichip average) algorithm was used for background correction, intra- and intermicroarray normalization, and expression signal calculation (Bolstad et al., 2003; Irizarry et al., 2003a,b). Once the absolute expression signal for each gene (i.e., the signal value for each probe-set) was calculated for the whole microarray set, the SAM (significance analysis of microarrays) algorithm (Tusher et al., 2001) was applied to calculate significant differential expression and to find the gene probesets that changed in each set of samples. The method uses permutations to provide robust statistical inference of the most significant probe sets and provides $p$ values adjusted to multiple testing, using the FDR (false discovery rate) (Benjamini and Hochberg, 1995). Different FDR cutoffs were applied to different comparisons during the study, keeping always a significance threshold of an FDR less than 0.3, which most of the time was less than 0.05 or less than 0.10 .

After identification of the differentially expressed gene probe-sets, the corresponding matrix of expression values for all performed microarray hybridizations was analyzed with the hclust (hierarchical cluster function) algorithm (Murtagh, 1985). This algorithm performs hierarchical cluster analysis with complete linkage to find similarity between gene probesets on the basis of their expression values along all the analyzed microarrays. The algorithm classifies gene probe-sets in correlated groups presenting similar expression profiles or expression signatures.

We applied all these methods using $\mathrm{R}$ and Bioconductor as main computational and bioinformatic tools (www. bioconductor.org; accessed October 2008). $\mathrm{R}$ is a programming language and software environment for statistical computing (www.r-project.org; accessed October 2008).

\section{Functional analysis}

Functional analysis of the significant genes obtained for each induced state was done with a functional annotation tool called GeneCodis (Gene Annotations Co-occurrence Discovery; see http://genecodis.dacya.ucm.es/) (CarmonaSaez et al., 2007). This tool allows the user to find combinations of co-occurrent annotations that are significantly associated with a list of genes under study with respect to a reference list. The significance of the annotations is calculated with a hypergeometric statistical test with FDR $p$ value correction and, using as reference, the mouse genome. The annotations were done at the same time with reference to the full Gene Ontology database (GO; http://www.geneontology.org/; accessed October 2008) and to the KEGG pathway database (KEGG; http://www.genome.ad.jp/kegg/ pathway.html; accessed October 2008). After the analyses done with GeneCodis the redundancy on the list of genes that are assigned to each functional class was depurated by manual curation.

\section{GFP expression}

Mice were injected with $75 \mu \mathrm{g}$ of $\mathrm{P}_{\mathrm{GFP} w p r e}$ by an HD procedure; 3 days later they were killed and livers were either perfused with collagenase solution (Sigma-Aldrich) to isolate hepatocytes as described previously (Berasain et al., 2005) or used to obtain sections that were frozen in O.C.T. compound (Tissue-Tek; Sakura Finetek, Torrance, CA). GFP expression in liver sections was visualized with an Eclipse E800 (Nikon, Tokyo, Japan) fluorescence microscope. The percentage of transfected cells was established by fluorescence-activated cell-sorting (FACS) analysis on isolated hepatocytes resuspended in phosphate-buffered saline (PBS), using a FACSCalibur analyzer (BD Biosciences, San Jose, CA) with CellQuest Pro software analysis.

\section{Measurement of plasma alanine aminotransferase and aspartate aminotransferase levels}

Plasma alanine aminotransferase (ALT) and aspartate aminotransferase (AST) levels were measured with a Hitachi 911 automatic analyzer (Hitachi, Tokyo, Japan). Values are expressed as international units per liter (IU/liter).

\section{Results \\ Determination of liver transfection efficiency and trans-activator expression}

The aim of our study was to evaluate the effects of constitutive expression of $\mathrm{rtTA}^{\mathrm{S}}$-M2 (rtTA2) or GLp65 in mouse liver by transfection of hepatocytes with plasmids expressing each of these TAs from a liver-specific promoter. To assess the efficiency of liver transfection by hydrodynamic (HD) injection we first injected mice with $75 \mu \mathrm{g}$ of a plasmid carrying GFP as a reporter gene $\left(\mathrm{P}_{\mathrm{GFP} w p r e}\right)$. We used this amount of plasmid because previous experiments performed with a plasmid encoding LacZ ( $\mathrm{pCMV} \beta$ ) had shown that maximal transfection efficiency was achieved with 50-100 $\mu \mathrm{g}$ of DNA (data not shown). Liver sections were examined by fluorescence microscopy at $72 \mathrm{hr}$ posttransfection, revealing that a significant amount of liver cells expressed the transgene (Fig. 1B). The percentage of in vivo-transfected hepatocytes was determined by isolating these cells after perfusion and subsequent analysis by flow cytometry, showing that $17 \%$ of hepatocytes were positive for GFP expression (Fig. 1C).

Plasmids EA-rtTA2 and EA-GLp65, carrying the rtTA2 ${ }^{\mathrm{S}}$ M2 (rtTA2) or GLp65 gene downstream from the liver-specific EalbPa1AT promoter, respectively, were constructed as described in Materials and Methods (Kramer et al., 2003) (Fig. 1A). To determine the level of expression for each TA, mice $(n=10)$ were injected by the HD procedure with $75 \mu \mathrm{g}$ of EA-rtTA2 or EA-GLp65 plasmid, respectively. One month 
A

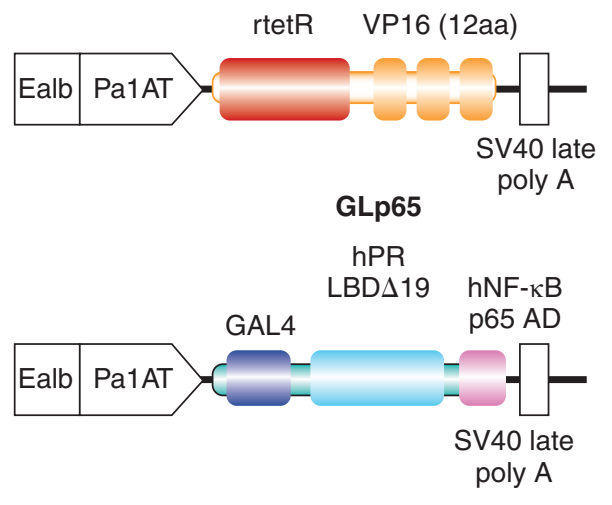

B

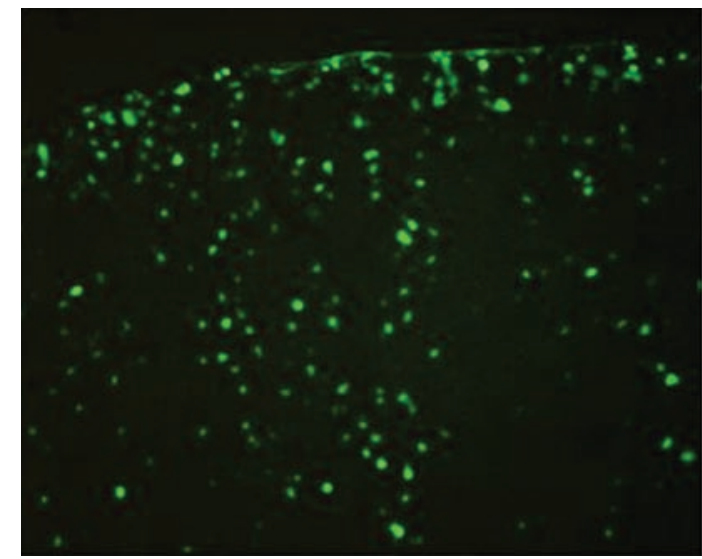

D

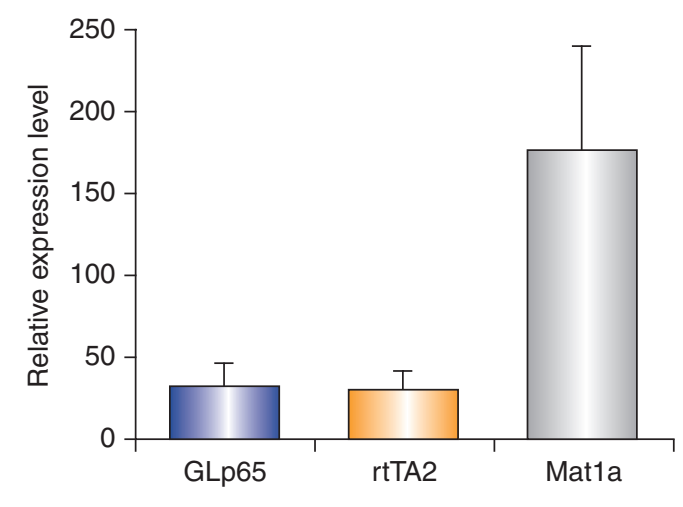

FIG. 1. Schematic representation of vectors expressing TAs and analysis of in vivo transfection and expression. (A) The EA-rtTA2 plasmid encodes rtTA2 ${ }^{\mathrm{s}}$-M2 TA, which is a fusion of the DNA-binding domain (DBD) of mutated bacterial tetracycline repressor (rtetR) with three repeats of the herpes simplex virus protein 16 (VP16) activation domain (AD). The EAGLp65 plasmid encodes GLp65 TA, which is a fusion of the yeast GAL4 DBD (GAL4), a mutated human progesterone receptor ligand-binding domain (hPR LBD $\Delta 19$ ), and the human NF- $\kappa$ B p65 subunit AD. Ealb, mouse albumin enhancer; Pa1AT, human $\alpha_{1}$-antitrypsin promoter; SV40 late poly A, polyadenylation signal from simian virus 40 (SV40). (B and C) Evaluation of in vivo transfection efficiency. BALB/c female mice were injected by a hydrodynamics (HD)-based procedure with $\mathrm{P}_{\mathrm{GFP}}$ wpre plasmid and 3 days later GFP expression was analyzed by fluorescence in liver sections (B) or by flow cytometry of hepatocytes isolated by liver perfusion (C). (D) Evaluation of TA expression in vivo. BALB/c female mice were injected by the HD procedure with EA-rtTA2 or EA-GLp65 plasmids and 30 days later liver TA expression was quantified by qRT-PCR. The graph shows the relative TA (rtTA2 or GLp65) transcript levels as compared with the endogenous liverspecific gene Mat1a. The expression level of each TA was normalized for $17 \%$ of hepatocytes (estimated amount of transfected hepatocytes), whereas Mat1a was normalized for $60 \%$ of liver cells (estimated amount of hepatocytes in liver). The histogram corresponds to a representative experiment.

later, rtTA2 or GLp65 liver expression was determined by quantitative RT-PCR (qRT-PCR) and compared with the expression level of Matla, which is a liver-specific gene. We found that expression of both TAs was similar and about 6fold lower than that of Mat1a, which is expressed at a medium to low level in hepatocytes (Fig. 1D). These data indicated that in our experimental setting both rtTA2 and GLp65 are expressed in a relatively high number of hepatocytes within physiological range levels.

\section{Global transcriptomic effect due to rtTA2 or GLp65 expression in liver}

To evaluate the constitutive effects of rtTA2 or GLp65 expression in the liver, groups of mice were injected by the HD procedure with $75 \mu \mathrm{g}$ of plasmid EA-rtTA2 or EA-GLp65. In each of these groups, half of the animals were treated with the inducer drug (Dox or RU-486, respectively). Similar groups of control noninjected animals were left untreated or received only the inducer drug (Dox or RU-486, respectively). One month after HD injection mice were killed and mRNA was isolated from livers and analyzed by microarrays to evaluate gene expression changes caused by rtTA2 and GLp65 TAs.

A total set of 21 mRNA samples from different mouse livers was used to hybridize the mouse genome 430A $2.0 \mathrm{mi}-$ croarrays (Affymetrix). The samples were as follows: (1) three controls, biological replicates of untreated mice; (2) three treated with rtTA2; (3) three treated with GLp65; (4) 
three treated with rtTA2 plus Dox; (5) three treated with GLp65 plus RU-486; (6) three treated only with the drug Dox; (7) three treated only with the drug RU-486.

To determine the effects of the TAs in the absence of the inducer drug (i.e., TA in the nonactive form) we compared the patterns of gene expression profile obtained from animals injected with plasmids encoding rtTA2 or GLp65 with those of untreated control mice. After normalization and expression signal calculation, the SAM algorithm was used to identify transcripts that showed a differential response between the compared groups (see Materials and Methods). Quantitative differences at the level of gene expression between treated and control animals are shown in a plot (Fig. 2). Animals expressing rtTA2 showed a moderate effect, as only 69 gene probe-sets were altered with an FDR of 0.05 , most of which were upregulated (Figs. 2A and 3). On the other hand, GLp65 had more pronounced effects on gene expression because 1059 gene probe-sets were altered with an FDR of 0.05 (Figs. 2D and 3). In this case the number of upregulated and downregulated probe-sets was similar and the higher slope deviation from the diagonal (compare Fig. 2D and A) indicates a more significant effect on the gene expression profiles. The number of altered gene probe-sets found in these comparisons using a more open FDR of 0.10 showed the same tendency: 1138 probe-sets for rtTA2 and 5716 probe-sets for GLp65.

To test the effect of each TA in the presence of its corresponding inducer drug (i.e., TA in the active form) we compared gene expression profiles of mice injected with the TAexpressing plasmid and treated with the inducer drug with those of mice injected with the TA plasmid without the drug. We found a small number of gene probe-sets altered either by rtTA2 plus Dox (35 probe-sets at an FDR of 0.05) (Fig. 3) or by GLp65 plus RU-486 (3 probe-sets at an FDR of 0.05) (Fig. 3), suggesting that most of the effects caused by the TA were already present in the absence of the inducer drug.

Regarding the effect of the drugs by themselves, we also compared gene expression profiles between control mice receiving Dox or RU-486, respectively, and untreated mice. The administration of Dox altered only 10 gene probe-sets at an FDR of 0.05 (14 at an FDR of 0.1) (Fig. 2C), mainly upregulated. RU-486 administration had more pronounced effects, causing differential expression of 95 gene probesets at an FDR of 0.05 (which increased to 314 at an FDR of 0.1 ), of which $37 \%$ were inhibited (Fig. 2F). A summary of the number of altered gene probe-sets observed at an FDR of 0.05 in each of the six contrasts mentioned is shown in Fig. 3.
A

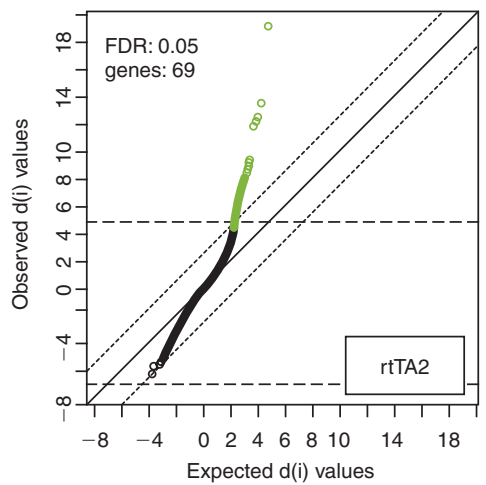

D

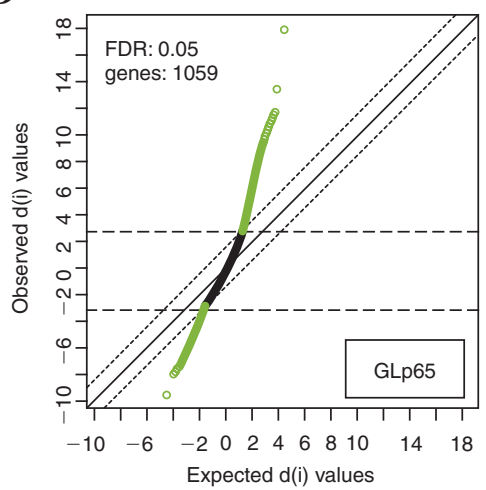

B

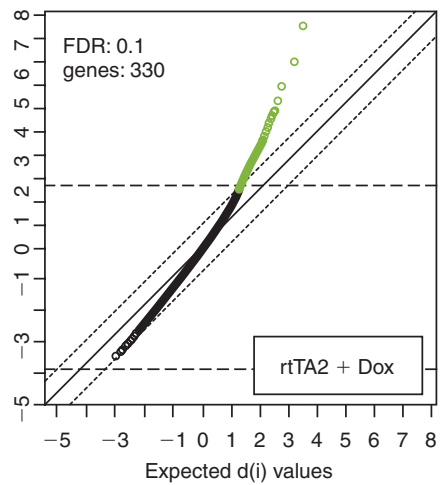

E

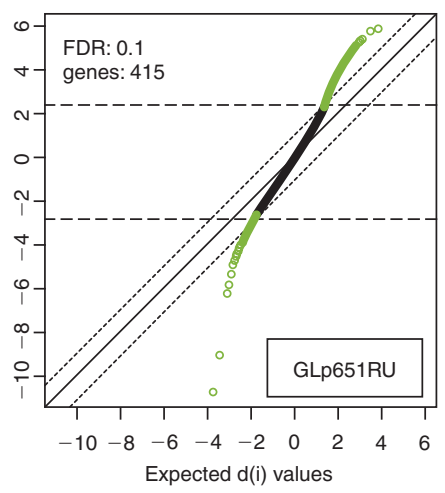

$\mathbf{C}$

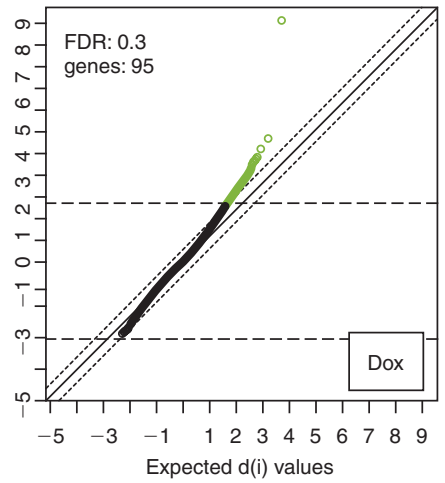

$\mathbf{F}$

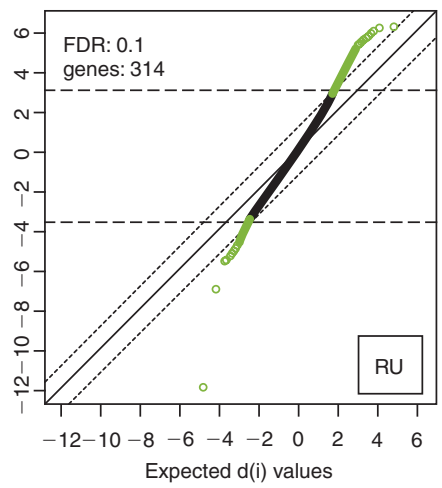

FIG. 2. Analysis of transcript differential response by means of the SAM algorithm. (A-C) Plots of experimental groups for the Tet-on system. (A) rtTA2, animals receiving EA-rtTA2 plasmid versus untreated animals; (B) rtTA2+Dox, animals receiving EA-rtTA2 plasmid and Dox versus animals receiving only EA-rtTA2; (C) Dox, animals receiving Dox versus untreated animals. (D-F) Plots of experimental groups for RU-486-on system. (D) GLp65, animals receiving EA-GLp65 plasmid versus untreated animals; (E) GLp65+RU, animals receiving EA-GLp65 plasmid and RU-486 versus animals receiving only EA-GLp65; (F) RU, animals receiving RU-486 versus untreated animals. Each point represents a single gene probe-set on the array. Diagonal data points (in black) correspond to nonsignificant changes in differential expression. Green data points correspond to gene probe-sets with significant change. 


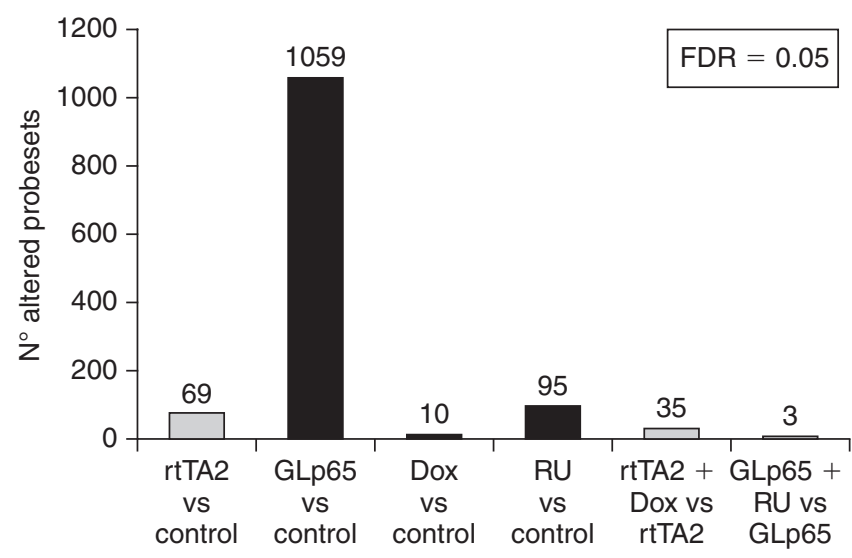

FIG. 3. Global transcriptomic effect of the Tet-on and RU486-on systems. To analyze the effect of TAs and their inducer drugs, comparisons of differential expression signals between the indicated groups were performed. The signal corresponds in all cases to an FDR less than 0.05 as cutoff.

As a means of establishing a more restrictive selection criterion to determine the number of altered genes in each treatment group we considered only those genes with an R-fold absolute value of $>3$ up- or downregulated (i.e., R-fold $\log _{2}$ : $>3$ for upregulated or $<0.33$ for downregulated). In the case of rtTA2, 25 gene probe-sets met this criterion (corresponding to 20 different genes), being all upregulated. From these, three genes corresponded to unknown genes that have not been annotated yet, leaving 17 unique known genes (see Table S1 at http://www.liebertonline.com/hum). GLp65 analysis provided 58 gene probe-sets altered (corresponding to 53 different genes), all of them upregulated. Only 45 of these were known, characterized genes (see Table S1). None of these genes showed an R-fold value $\geq 10$. The comparison of these two lists (see Table S1) shows that the top 17 genes altered by rtTA2 are also altered by GLp65, indicating that both TAs have a similar gene alteration profile, at least in the most significant region. The data also showed that GLp65 provokes larger effects.

\section{Microarray data validation}

As the next step of the study, we focused on gene expression changes observed in animals treated only with the inducer drugs, because these drugs have been widely used and their side effects have been described extensively in the literature. Moreover, the effects of drug intake should be appreciated in all liver cells, which facilitates the validation of changes observed.

Three lines of evidence supported the authenticity of the changes observed in mice treated with the inducer drugs. First, the internal coherence of the microarray was confirmed when comparing data obtained with several probe-sets corresponding to the same differentially expressed gene. As shown in Fig. 4A and B, multiple probe-sets corresponding to representative genes displayed the same alteration direction with a similar fold change, indicating a high degree of consistency for our analysis. Second, when four representative genes altered in each group (Dox or RU-486, respectively) were analyzed by qRT-PCR the results confirmed not only a strongly induced gene (Orm2), but also some other genes induced moderately in the microarrays but clearly observed by PCR (such as Col1a2 and Saa3; see Fig. 4C and D). The fold changes determined by PCR for these genes were also consistent with the fold changes determined by the microarrays. Finally, the information previously recorded in the literature about the effects associated with the administration of Dox and RU-486 correlates well with changes observed in this study. These changes include the upregulation of several genes of the collagen family (Col1a1, Col1a2, Col3a1, Col5a2, and Col6a2) in animals treated with Dox (see Table S2 at http://www.liebertonline.com/hum), which correlates with the profibrotic activity of this antibiotic (Lee et al., 2003; Sapadin and Fleischmajer, 2006). In the case of RU-486 we observed alteration of genes implicated in immunological functions in animals treated with this drug (Bcan, Cfh, C1r, F13b, and several interleukin receptors: Il1rn, Il10rb, and Il13ra1) (see Table S3 at http://www.liebertonline.com/hum). These findings are in keeping with the property of RU-486 to depress the innate immune system (Miech, 2005). It is also known that RU-486 acts on steroid receptors, and we observed several glucocorticoid and steroid hormone receptors altered in RU-486-treated animals (Nr1d2 and Nr3c1) (SitrukWare, 2006).

\section{Functional categorization of microarray data}

To generate a functional profile for the genes altered by the expression of each TA in mice we have used GeneCodis, which is a Web-based tool for the ontological analysis of large lists of genes (see Materials and Methods) (Carmona-Saez et al., 2007). The functional profile constructed by GeneCodis for the 68 significant gene probesets (60 genes) altered by rtTA2 (for an FDR of about 0.05 ) (Table 2) revealed that the most significant functional alterations corresponded to some genes involved in apoptosis (Birc4, Casp3, Cflar, and Hipk2) and negative regulation of cell cycle (Casp3, Hipk2, and Rb1cc1). Other functions in which altered genes were found with lower significance included translation initiation (Eif3s8, Eif3s10, and Eif4g1). In the case of genes altered by GLp65, the 1058 significantly altered probe-sets (for an FDR of about 0.05) corresponded mainly to genes involved in apoptosis (Birc4, Casp3, Cflar, and Hipk2) and transport (Abca1, Abcb7, Abcc2, and Abcd2) (see Table 2). Relevant pathways in which GLp65 altered genes are implicated included mitogen-activated protein kinase (MAPK) signaling pathway and ATP-binding cassette (ABC) transporters.

A functional analysis of genes altered by each TA in their induced stage was also performed (taking genes with an FDR cutoff of 0.1). According to GeneCodis, genes differentially altered between the rtTA2 plus Dox group and the rtTA2 group corresponded, with a high degree of statistical probability, to collagen molecules implicated in cell adhesion, acute-phase response proteins (Orm1, Orm2, Saa1, Saa2, and Saa3), and lipid transporter activity associated with these proteins (see Table S4 at http://www.liebertonline.com/hum). Genes differentially expressed between the GLp65 plus RU486 group and the GLp65 group appeared to be implicated in pathways related to regulation of actin cytoskeleton and adherens junction (Actn4, Iqgap1, Mapk1, and Wasl). In addition, transcriptional activator activities were also signifi- 
A

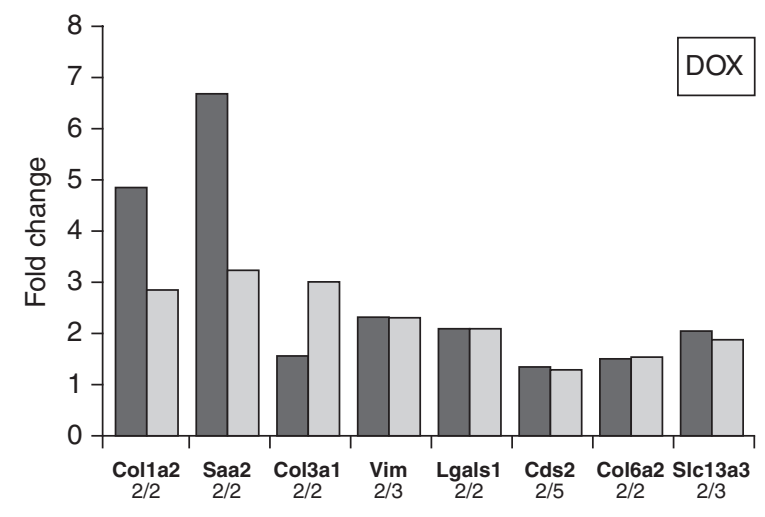

B

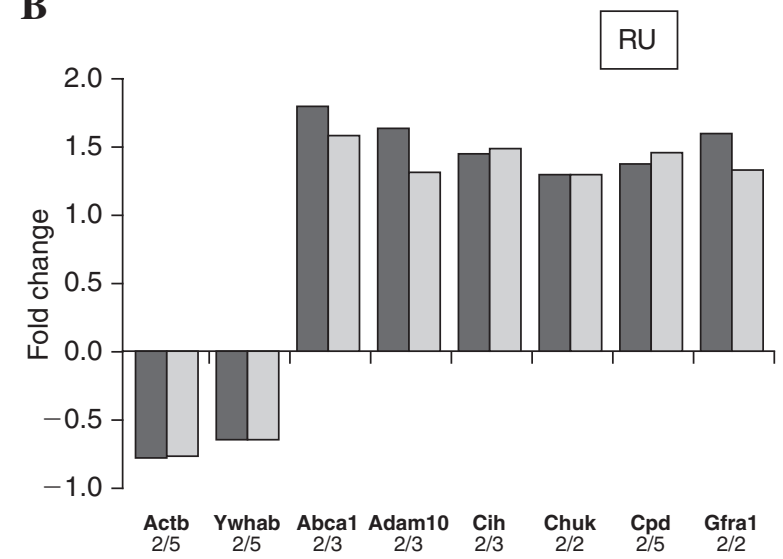

C

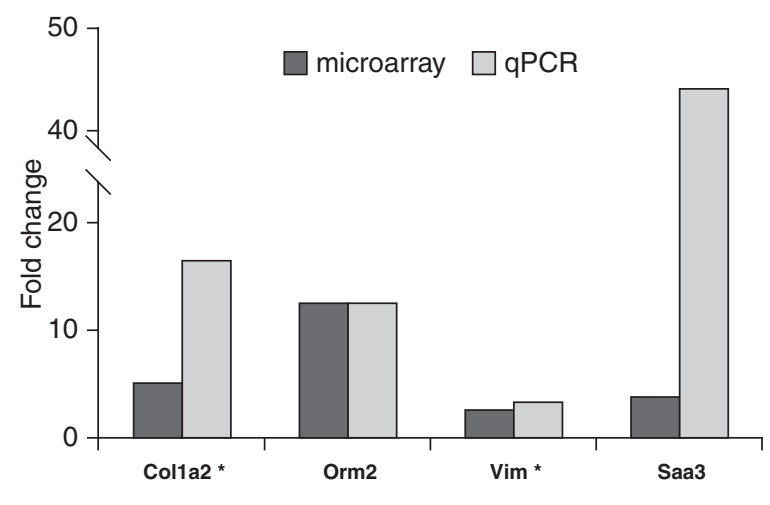

D

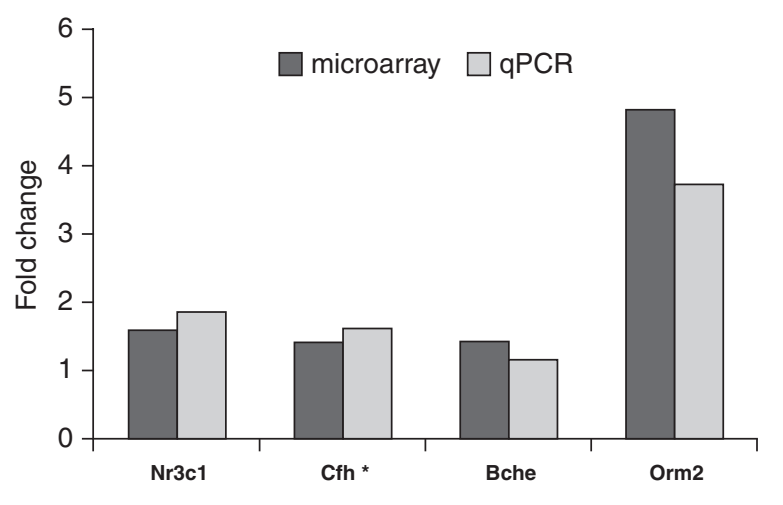

FIG. 4. Validation of microarray data in mice receiving the inducer drugs. Comparison of the R-fold expression values obtained by microarray analysis with redundant probe-sets for eight representative genes differentially expressed in (A) Dox-treated mice and (B) RU-486-treated mice. Data from two probe-sets showing altered expression for each analyzed gene are shown (shaded and solid columns). The number of specific probe-sets showing altered expression versus the total number of probe-sets present in the microarray for each validated gene is shown below the gene name. (C and D) Comparison of the R-fold expression values obtained by microarray analysis and by qRT-PCR for four representative genes differentially expressed in (C) Dox-treated mice and (D) RU-486-treated mice. *Genes validated both by several probe-sets in the microarrays and by qRT-PCR.

cantly altered (Fus, Ppargc1a, and Sfpi1) in this group (see Table S5 at http://www.liebertonline.com/hum).

To understand whether the effect of the TAs in the presence of the inducer drug was due to changes in the TA or to the drug itself, data obtained from animals after drug administration were also analyzed with the same functional annotation tool. GeneCodis analysis of the 95 probe-sets altered (86 genes) after Dox administration (using an FDR cutoff of 0.3) revealed a significant presence of two families of genes involved in the acute-phase response (Orm1, Orm2, Orm3, Saa1, Saa2, and Saa3). The consistent appearance of five collagen genes (Col1a1, Col1a2, Col3a1, Col5a2, and Col6a2) and four genes related to membrane transporter activity (Scl6a8, Scl6a12, Scl13a3, and Scl22a7) is also significant (see Table S2). This correlates well with the main effect described for active rtTA2, strongly suggesting that the additional effects observed when rtTA2 is activated by Dox are due mainly to the drug itself, and not to a change in rtTA2. GeneCodis analysis of data obtained from the RU-486- treated animals showed a functional effect less focused on specific gene families; rather, the effect was broader, affecting biological processes more general and with a significant effect on signal transduction and cell communication (see Table S3), including some protein kinases and phosphatases (Csnk2a1, Mapk, Ppp1cb, and Prkar2a). Alteration of these genes shows that this drug may produce harsher physiological effects. These processes were also significantly altered in the GLp65 plus RU-486 group, suggesting that most of the additional effects observed when GLp65 is activated by RU486 are also due to the drug itself.

\section{Microarray data clustering}

To achieve a more open and integrative analysis of the transcriptomic profiles due to the specific effect of each TA, the expression data corresponding to genes that change at level of significance FDR $\leq 0.10$ in animals treated with rtTA2 plus Dox and with GLp65 plus RU-486 (Fig. 2B and 


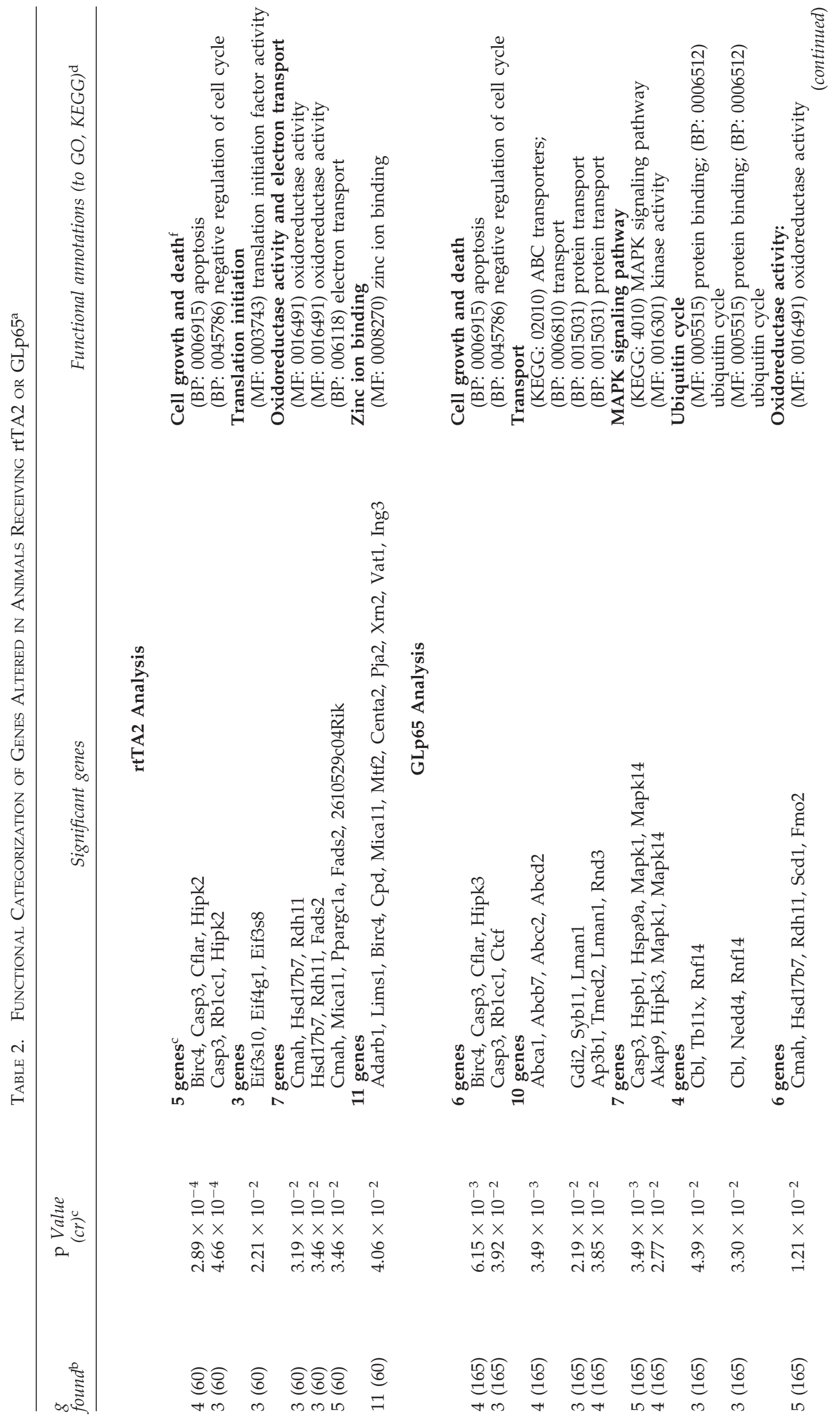




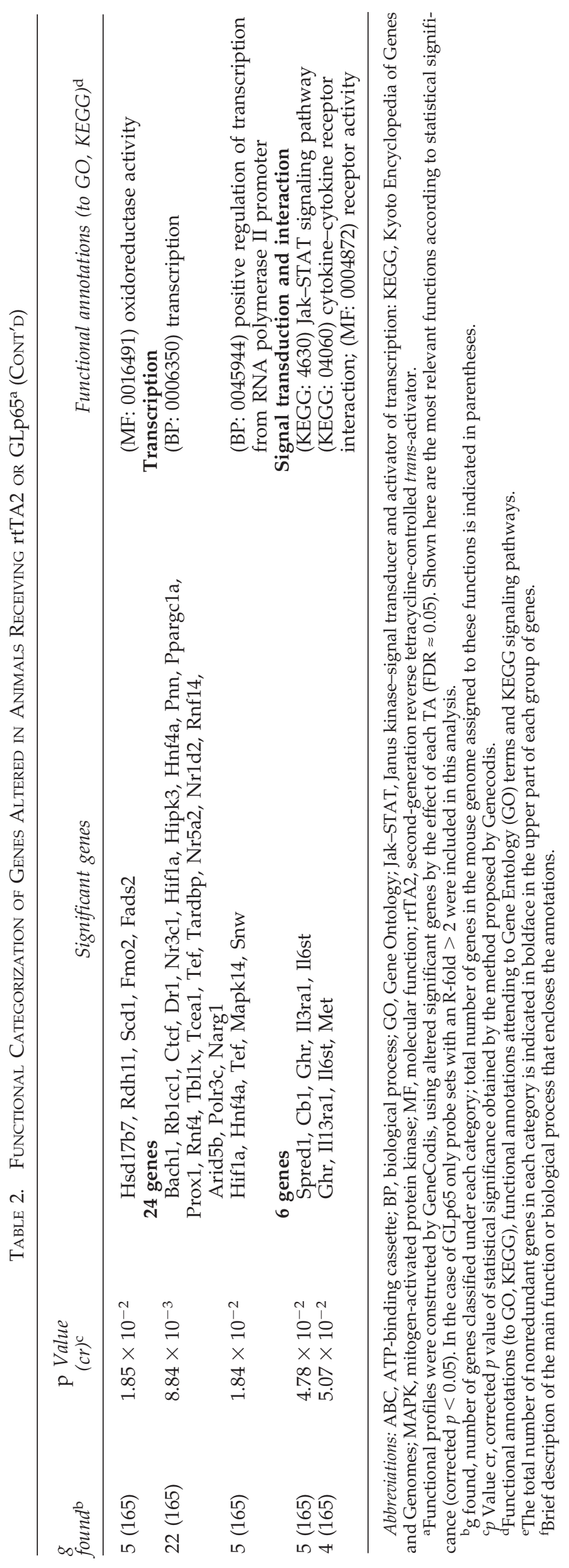




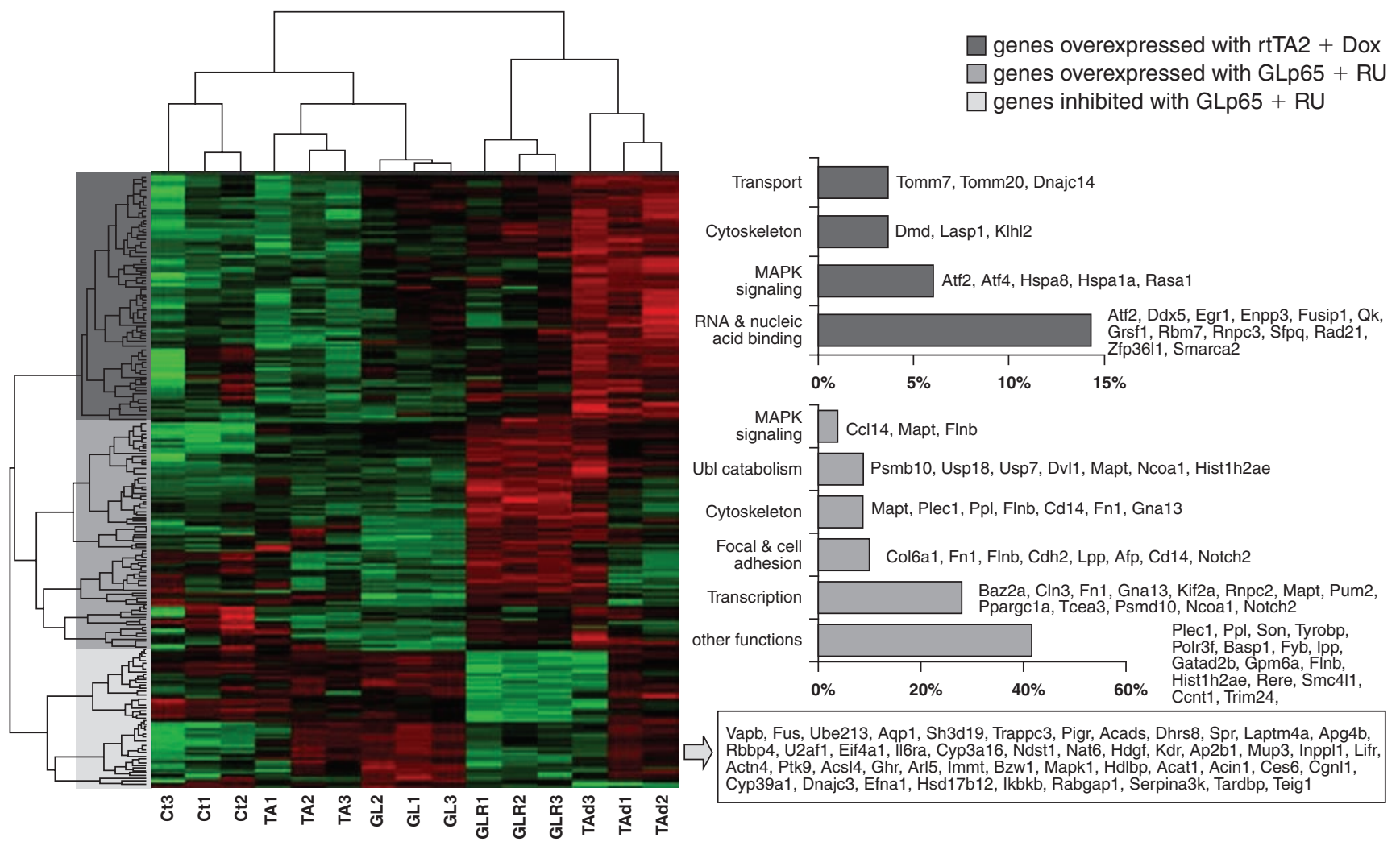

FIG. 5. Hierarchical clustering of experimental groups receiving the TA proteins. Probe-sets found altered in the presence of each TA plus inducer drug combination were used $(p<0.001)$ after eliminating those present in the drug contrasts. The heat map presents a grid of colored points, with each color representing a gene expression value in the sample: red corresponding to high expression, green corresponding to low expression, and black corresponding to an intermediate level of expression. Each column represents a sample and rows represent altered genes. The left margin dendrogram indicates three distinct clusters of genes. Graphics on the right margin show the results of GeneCodis analysis of two clusters of genes that are upregulated. The percentage of genes has been calculated with respect to the number of genes in each cluster. Ct, untreated; TA, rtTA2 treated; GL, GLp65 treated; Tad, mice receiving rtTA2 plus Dox; GLR, mice receiving GLp65 plus RU-486.

E) were taken and combined after subtracting genes altered by the effect of the inducer drug alone. From these pools we further selected the most significant genes, using a cutoff of $p \leq 0.001$ (i.e., gene-specific, differential expression adjusted $p \leq 0.001)$. In this way, we identified a list of 223 altered gene probe-sets that were used to explore the expression profiles and patterns of samples and genes.

The analysis was done by means of semisupervised hierarchical clustering, producing a heat map with the gene expression values of these 223 altered genes (see Fig. 5). With respect to the samples, this analysis provided a clear clustering of the biological replicates and the segregation of the five sample types including a clear separation of the TAs with and without the drug inducer (see top dendrogram in Fig. 5). These results indicate reproducibility of the gene expression data obtained by multiple microarray experiments. With respect to the genes, the heat map gives the overall view that 223 genes group in three distinct clusters: the first cluster is composed of 90 genes that are overexpressed in animals treated with rtTA2 plus Dox (TAd1, -2, and -3); the second cluster includes 83 genes overexpressed in animals treated with GLp65 plus RU-486 (GLR1, -2, and -3); and the third cluster contains 50 repressed genes also in this group. Finally, considering that the genes altered only in response to the drugs have been filtered out from this heat map, the overall biological-functional trend of the overexpressed genes showed similar results to those recorded for TA plus inducer analysis (see Tables S5 and S6), with a larger effect observed for GLp65 because it includes alterations in the process of transcription, not present in the case of rtTA2. Of all genes overexpressed in the presence of rtTA2 plus Dox (see the heat map in Fig. 5) there was only one (Rasa1) also overexpressed in the presence of rtTA2 without drug, which means that the expression of all remaining 89 genes was due only to the effect of rtTA2 in its active stage. However, this is considering FDR $\leq 0.10$ as threshold; when taking as a level of significance FDR $\leq 0.05$ (as indicated in Fig. 3) only two of these genes (Dnajc14 and Atf4) would be included in the heat map. In the same way, none of the genes overexpressed by the effect of GLp65 plus RU-486 included in the heat map could be considered when using FDR $\leq 0.05$, and only three downregulated genes (Fus, Spr, and Vapb) would be significant for this FDR value. Therefore, despite the interest of the functional assignment done for the genes of the heat map (Fig. 5), the overall effect of the TAs can be considered mild because it is not significant.

\section{Evaluation of toxicity after constitutive expression of TAs in liver}

Alanine aminotransferase (AST [SGOT]) and aspartate aminotransferase (ALT [SGPT]), which are sensitive indica- 

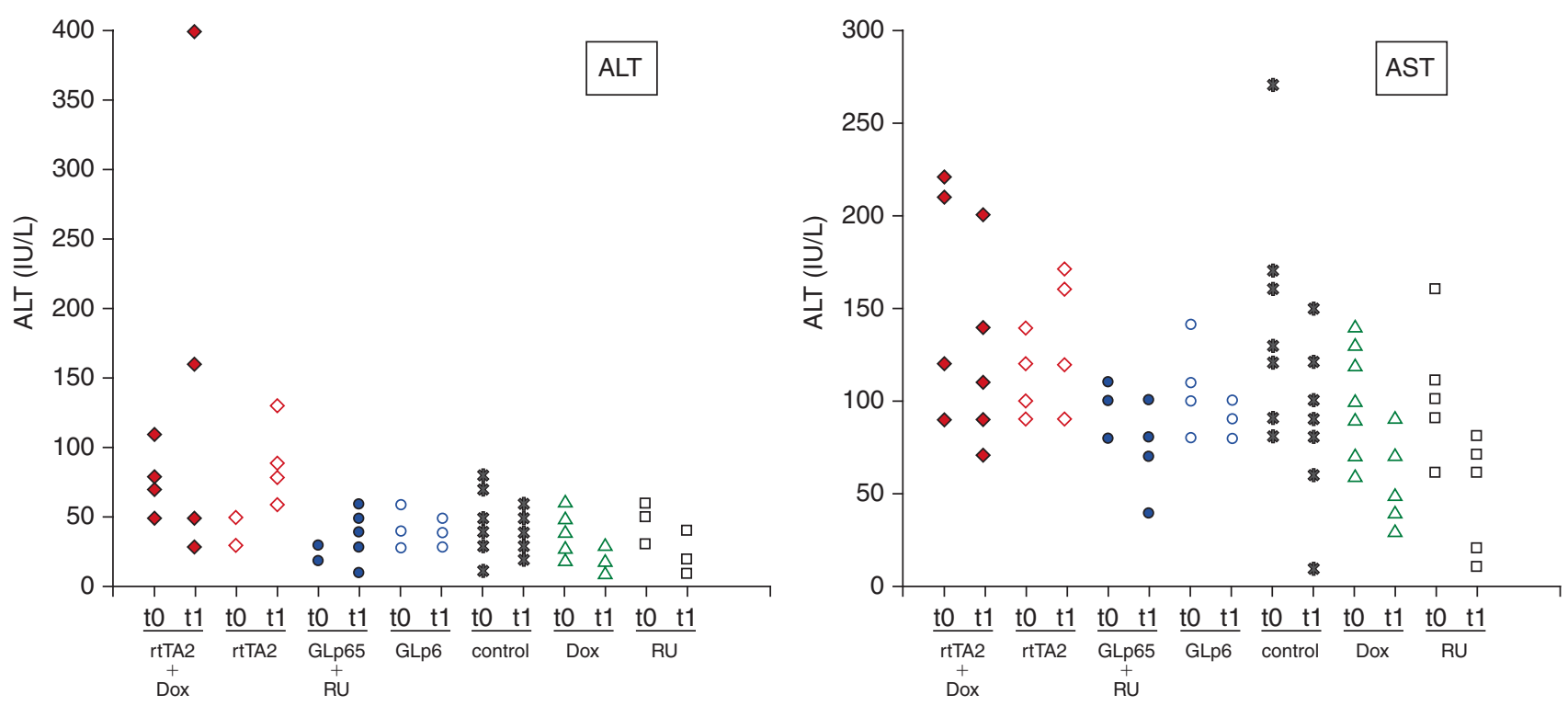

FIG. 6. Analysis of toxicity. Alanine aminotransferase (ALT) and aspartate transferase (AST) levels were analyzed in sera of mice from all experimental groups before treatment $(\mathrm{t} 0)$ and 30 days later before sacrifice (t 1$)$. Control, mice that underwent no treatment $(n=7)$; Dox and RU, mice that received only the drug $(n=7)$; rtTA2 and GLp65, mice that received only the TA $(n=5)$; rtTA2+Dox and GLp65+RU, mice that received the TA and its corresponding drug $(n=5)$. The value for each individual mouse is plotted.

tors of liver damage, were measured in samples of mouse sera collected on day 0 (before plasmid injection) and day 30 (just before sacrifice). As shown in Fig. 6 no relevant changes in ALT or AST levels were observed in any of the various groups 1 month after treatment, with the exception of one animal, from the rtTA2 plus Dox group, which showed a moderate increase in ALT level.

Liver sections from animals belonging to the various groups were stained with hematoxylin-eosin and analyzed in order to determine whether any structural damage had taken place. No relevant liver damage was observed by light microscopy (data not shown).

\section{Discussion}

Gene-regulable systems have been used to activate or suppress the expression of transgenes encoded by long-term expression vectors. This is essential to adapt transgene expression to therapeutic needs and to avert the toxicity derived from inappropriate levels of the encoded therapeutic molecule. These regulable systems are based on the use of a TA protein (expressed from a constitutive promoter), which only in the presence of a specific drug (inducer) is able to bind to the minimal inducible promoter that controls the expression of the therapeutic transgene. Although TAs are usually expressed at low levels, the fact that they are continuously present in the transduced cell could result in toxicity due to the activation or inactivation of nontarget genes. The goal of the present study was to analyze the effects of constitutive expression of the TAs from Tet-on and RU486on systems (rtTA2 and GLp65, respectively) on gene expression profiles in mouse liver. These two TAs were chosen because they have been extensively used to regulate expression of therapeutic genes in animal models and could have a potential use in human patients (Wang et al., 2004; Zabala et al., 2004). Therefore, analysis of their possible toxicity in vivo is essential before they can be used in gene therapy clinical protocols.

To achieve TA expression in a high percentage of hepatocytes HD injection was used as an efficient method to achieve efficient and durable transduction of liver cells (Liu et al., 1999; Hodges and Scheule, 2003). Although this technique is distant from clinical applications we decided to employ it because the use of viral or nonviral vectors would introduce additional components, such as viral proteins or synthetic transducing agents, that could induce an undesired toxicity or/and immunogenic reaction, making it more difficult to determine the specific effects induced by the trans-activators themselves.

We have previously shown that HD injection allowed sustained protein expression over at least 50 days in mice receiving a plasmid containing the human $\alpha_{1}$-antitrypsin (hAAT) gene controlled by the same promoter used in this study (Kramer et al., 2003). In addition, experiments performed in our laboratory showed that the amount of plasmid DNA transfected into mouse liver by hydrodynamic injection remains constant between 7 and 120 days (our unpublished data). Because transduction efficiency achieved in the present study was about $20 \%$, changes observed in gene expression profiles are most likely attenuated with respect to the changes taking place in the transfected cells, because whole liver was employed for transcriptome analysis. However, this level of transduction was comparable to that observed in gene therapy protocols based on delivery of adenoviral vectors to the liver, indicating that the observed changes will probably resemble those expected in a clinical setting with a similar degree of transfection. Although other liver cells apart from hepatocytes could also have been 
transfected in our study, we believe that the observed changes correspond mainly to hepatocytes because both plasmids that were used contain a liver-specific promoter (EalbPa1AT), restricting expression to these cells. Transcriptomic changes were analyzed 1 month after HD injection because we considered it a length of time long enough to discard possible side effects due to the transduction technique.

The effects produced by the drugs were more general, affecting theoretically $100 \%$ of liver cells, which facilitates the validation of the observed changes. Tetracyclines have been reported to show some biological properties besides their antibiotic effects, such as profibrogenic activity (Sapadin and Fleischmajer, 2006). As an example, Dox has been extensively used to inhibit collagenase in the treatment of periodontitis, even though its mechanisms of action are not completely understood (Sapadin and Fleischmajer, 2006). On the other hand, in vitro studies have shown that Dox administration induces more collagen expression than transforming growth factor- $\beta_{2}$ on rabbit mesothelial cells (Lee et al., 2003). Our microarray data showed that in the presence of Dox procollagen-1 and other genes of the collagen family (Col1a2, Col1a1, Col6a2, etc.) were upregulated, which correlates well with the described effects of Dox on collagen synthesis. Mifepristone (RU-486) is a synthetic steroid with potent antiprogesterone and antiglucocorticoid activities, being able to upregulate glucocorticoid receptors in the endometrium when used as a contraceptive (Narvekar et al., 2006). In addition to its other side effects, RU-486 has also been described as depressing the innate immune system (Miech, 2005). In agreement with these data, our microarray showed that in mice receiving RU-486 there was both an upregulation of several steroid receptors, such as $\mathrm{Nr} 3 \mathrm{c} 1$ and $\mathrm{Nr} 1 \mathrm{~d} 2$, and an alteration in a significant number of genes implicated in immunological processes. Changes in representative genes altered in the Dox and RU-486 groups, such as Colla2 and $\mathrm{Nr3c1}$, were also confirmed by RT-PCR analysis. Overall cluster analysis showed a coherent behavior of the data, with clear distinction and segregation of the samples of each group. Finally, the fact that animals belonging to each experimental group were easily clustered together when analyzed by heat mapping supports the validity of the data.

In general, the data obtained from the microarray analysis of animals expressing either rtTA2 or GLp65 in liver showed a moderate effect on gene expression profiles. The observed transcriptomic effect of rtTA2 versus control animals was weak, considering that is a genome-wide analysis and alterations were observed for a reduced number of genes (69 probe-sets with a significance FDR of 0.05). On the other hand, GLp65 had a more pronounced effect on gene expression because 1059 probe-sets were altered with an FDR of 0.05 . In this analysis only 20 genes from the rtTA2 group and 53 genes from the RU-486 group showed an alteration with an R-fold $\geq 3$, indicating that constitutive expression of these TAs in the liver induce only moderate changes in the transcriptome profile.

Our functional analysis showed that apoptosis and negative regulation of cell cycle were the functions more affected in both the rtTA2 and GLp65 groups, with a similar pattern of altered genes (see Table 2). However, despite these transcriptional changes the immunohistochemical analysis of liver samples from mice expressing rtTA2 or GLp65 showed no increase in apoptosis (data not shown) In this particular case, although func- tional analysis revealed a group of four altered genes involved in apoptosis (BIRC4, CASP3, CFLAR, and, HIPK2) (see Table 2), only one of them (BIRC4) showed an R-fold value greater than 3 (see Table S1), indicating that these changes might not be strong enough to cause manifest phenotypic alterations.

Other functions that were altered, with lower significance, were translation initiation activities in rtTA2-expressing animals and ABC transport and MAPK signaling in the GLp65 group. Mapk1 and Mapk14 overexpression was confirmed by qRT-PCR in this group (data not shown), indicating that this pathway may be affected by GLp65.

It was also important to analyze the global transcriptomic effect induced by the TAs when they were in their active form. To simplify this analysis we compared animals expressing rtTA2 or GLp65 and receiving Dox or RU-486, respectively, with those animals that expressed only the corresponding TA. This comparison revealed that the active drugs induced only discrete additional changes in gene expression with respect to their nonactive forms (35 altered probe-sets for rtTA2 plus Dox and only 3 inhibited probesets for GLp65 plus RU-486, with an FDR of $\sim 0.05)$. Most changes observed in these contrasts were in fact due to the effects produced by the inducer drugs by themselves. As noted previously, in the case of rtTA2 plus Dox the most relevant changes were those observed in the procollagen family $(\mathrm{Col})$ of structural proteins and in acute-phase proteins of the serum amyloid (Saa) and orosomucoid (Orm) families, which were also highly significant in mice receiving only Dox. Both the moderate transcriptomic effects and the absence of liver toxicity induced by rtTA2 plus Dox are in agreement with studies demonstrating the possibility of establishing stable hepatocyte-derived cell lines expressing rtTA2 (Goldring et al., 2006). These results are in contrast with the adverse effects described previously in cells expressing tTA, which is the repressor version of rtTA (Gossen and Bujard, 1992; Gallia and Khalili, 1998). However, in this case these toxic effects were most likely associated with the VP16 AD present in tTA, which has been reduced in the rtTA2 version. Moreover, in such studies tTA was expressed from a promoter that could be activated by tTA itself, which probably resulted in high levels of the activated TA, producing morphological changes and alterations in the cell cycle. In contrast, when tTA was expressed from a CMV promoter, which is stronger than the EalbPa1AT promoter used in the present study, no deleterious effects on cell cycle were observed in transfected cells (Qin and Barsoum, 1997). An additional piece of data supporting the lack of toxicity of the TAs used in this study is that both Tet-on and Gal-4-based regulatory systems have been successfully used to generate transgenic mice (for a review see Lewandoski, 2001).

In summary, our findings demonstrate that the expression of rtTA2 or GLp65 in liver of treated mice produces a modest transcriptomic effect, which is not significantly enhanced when the inducer drug is present. These results, together with the absence of toxicity observed in treated animals, indicate that Tet-on and RU486-on could constitute gene-regulatory systems with a good degree of safety for liver expression. The genome-wide view given by the microarrays provides specific indication of which biological functions are most affected and this view will help to determine any further use or evaluation of these gene regulatory systems. However, further toxicity analysis should be performed in 
nonhuman primates before applying these systems in human clinical protocols.

\section{Acknowledgments}

The authors thank M. Zabala (CIMA) for performing hydrodynamic injections and R. Hernandez-Alcoceba for critical reading of the manuscript. This work was supported by grant FIS (PI042108) and grant C03/02 (Instituto Carlos III), and through the UTE Project CIMA. CIBERehd is funded by the Instituto de Salud Carlos III. Dr. J. De Las Rivas also acknowledges support provided by FIS grant PI061153.

\section{Author Disclosure Statement}

No competing financial interests exist.

\section{References}

Antonsson, A., Hughes, K., Edin, S., and Grundstrom, T. (2003). Regulation of c-Rel nuclear localization by binding of $\mathrm{Ca}^{2+}$ /calmodulin. Mol. Cell. Biol. 23, 1418-1427.

Baron, U., Gossen, M., and Bujard, H. (1997). Tetracycline-controlled transcription in eukaryotes: Novel transactivators with graded transactivation potential. Nucleic Acids Res. 25, 2723-2729.

Benjamini, Y., and Hochberg, Y. (1995). Controlling the false discovery rate: A practical and powerful approach to multiple testing. J. R. Stat. Soc. 57, 289-300.

Berasain, C., Garcia-Trevijano, E.R., Castillo, J., Erroba, E., Santamaria, M., Lee, D.C., Prieto, J., and Avila, M.A. (2005). Novel role for amphiregulin in protection from liver injury. J. Biol. Chem. 280, 19012-19020.

Blau, J., Xiao, H., McCracken, S., O'Hare, P., Greenblatt, J., and Bentley, D. (1996). Three functional classes of transcriptional activation domain. Mol. Cell. Biol. 16, 2044-2055.

Bolstad, B.M., Irizarry, R.A., Astrand, M., and Speed, T.P. (2003). A comparison of normalization methods for high density oligonucleotide array data based on variance and bias. Bioinformatics 19, 185-193.

Burcin, M.M., Schiedner, G., Kochanek, S., Tsai, S.Y., and O'Malley, B.W. (1999). Adenovirus-mediated regulable target gene expression in vivo. Proc. Natl. Acad. Sci. U.S.A. 96, 355-360.

Candau, R., Moore, P.A., Wang, L., Barlev, N., Ying, C.Y., Rosen, C.A., and Berger, S.L. (1996). Identification of human proteins functionally conserved with the yeast putative adaptors ADA2 and GCN5. Mol. Cell. Biol. 16, 593-602.

Carmona-Saez, P., Chagoyen, M., Tirado, F., Carazo, J.M., and Pascual-Montano, A. (2007). GENECODIS: A web-based tool for finding significant concurrent annotations in gene lists. Genome Biol. 8, R3.

Espinosa, L., Ingles-Esteve, J., Robert-Moreno, A., and Bigas, A. (2003). I $\kappa \mathrm{B} \alpha$ and p65 regulate the cytoplasmic shuttling of nuclear corepressors: Cross-talk between Notch and NF $\kappa$ B pathways. Mol. Biol. Cell 14, 491-502.

Gallia, G.L., and Khalili, K. (1998). Evaluation of an autoregulatory tetracycline regulated system. Oncogene 16, 1879-1884.

Goldring, C.E., Kitteringham, N.R., Jenkins, R., Lovatt, C.A., Randle, L.E., Abdullah, A., Owen, A., Liu, X., Butler, P.J., Williams, D.P., Metcalfe, P., Berens, C., Hillen, W., Foster, B., Simpson, A., McLellan, L., and Park, B.K. (2006). Development of a transactivator in hepatoma cells that allows expression of phase I, phase II, and chemical defense genes. Am. J. Physiol. Cell. Physiol. 290, C104-C115.
Gossen, M., and Bujard, H. (1992). Tight control of gene expression in mammalian cells by tetracycline-responsive promoters. Proc. Natl. Acad. Sci. U.S.A. 89, 5547-5551.

Hall, D.B., and Struhl, K. (2002). The VP16 activation domain interacts with multiple transcriptional components as determined by protein-protein cross-linking in vivo. J. Biol. Chem. 277, 46043-46050.

Harvey, D.M., and Caskey, C.T. (1998). Inducible control of gene expression: Prospects for gene therapy. Curr. Opin. Chem. Biol. 2, 512-518.

Hodges, B.L., and Scheule, R.K. (2003). Hydrodynamic delivery of DNA. Expert Opin. Biol. Ther. 3, 911-918.

Irizarry, R.A., Bolstad, B.M., Collin, F., Cope, L.M., Hobbs, B., and Speed, T.P. (2003a). Summaries of Affymetrix GeneChip probe level data. Nucleic Acids Res. 31, e15.

Irizarry, R.A., Hobbs, B., Collin, F., Beazer-Barclay, Y.D., Antonellis, K.J., Scherf, U., and Speed, T.P. (2003b). Exploration, normalization, and summaries of high density oligonucleotide array probe level data. Biostatistics 4, 249-264.

Kramer, M.G., Barajas, M., Razquin, N., Berraondo, P., Rodrigo, M., Wu, C., Qian, C., Fortes, P., and Prieto, J. (2003). In vitro and in vivo comparative study of chimeric liver-specific promoters. Mol. Ther. 7, 375-385.

Lee, Y.C., Lane, K.B., Zoia, O., Thompson, P.J., Light, R.W., and Blackwell, T.S. (2003). Transforming growth factor- $\beta$ induces collagen synthesis without inducing IL-8 production in mesothelial cells. Eur. Respir. J. 22, 197-202.

Leonhardt, S.A., and Edwards, D.P. (2002). Mechanism of action of progesterone antagonists. Exp. Biol. Med. (Maywood) 227, 969-980.

Lewandoski, M. (2001). Conditional control of gene expression in the mouse. Nat. Rev. Genet. 2, 743-755.

Liu, F., Song, Y., and Liu, D. (1999). Hydrodynamics-based transfection in animals by systemic administration of plasmid DNA. Gene Ther. 6, 1258-1266.

Melero, I., Mazzolini, G., Narvaiza, I., Qian, C., Chen, L., and Prieto, J. (2001). IL-12 gene therapy for cancer: In synergy with other immunotherapies. Trends Immunol. 22, 113-115.

Miech, R.P. (2005). Pathophysiology of mifepristone-induced septic shock due to Clostridium sordellii. Ann. Pharmacother 39, 1483-1488.

Murtagh, F. (1985). Multidimensional Clustering Algorithms: COMPSTAT Lectures, No. 4 (Physica-Verlag, Wuerzburg, Germany).

Narvekar, N., Critchley, H.O., Cheng, L., and Baird, D.T. (2006). Mifepristone-induced amenorrhoea is associated with an increase in microvessel density and glucocorticoid receptor and a decrease in stromal vascular endothelial growth factor. Hum. Reprod. 21, 2312-2318.

Parry, G.C., and Mackman, N. (1997). Role of cyclic AMP response element-binding protein in cyclic AMP inhibition of NF- $\kappa$ B-mediated transcription. J. Immunol. 159, 5450-5456.

Prieto, J., Qian, C., Hernandez-Alcoceba, R., GonzalezAseguinolaza, G., Mazzolini, G., Sangro, B., and Kramer, M.G. (2004). Gene therapy of liver diseases. Expert Opin. Biol. Ther. 4, 1073-1091.

Qin, X.Q., and Barsoum, J. (1997). Differential cell cycle effects induced by E2F1 mutants. Oncogene 14, 53-62.

Sapadin, A.N., and Fleischmajer, R. (2006). Tetracyclines: Nonantibiotic properties and their clinical implications. J. Am. Acad. Dermatol. 54, 258-265.

Schmitz, V., Wang, L., Barajas, M., Gomar, C., Prieto, J., and Qian, C. (2004). Treatment of colorectal and hepatocellular carcinomas by adenoviral mediated gene transfer of endo- 
statin and angiostatin-like molecule in mice. Gut 53, 561-567.

Siebenlist, U., Franzoso, G., and Brown, K. (1994). Structure, regulation and function of NF- $\kappa$ B. Annu. Rev. Cell. Biol. 10, 405-455.

Sitruk-Ware, R. (2006). Mifepristone and misoprostol sequential regimen side effects, complications and safety. Contraception $74,48-55$.

Tusher, V.G., Tibshirani, R., and Chu, G. (2001). Significance analysis of microarrays applied to the ionizing radiation response. Proc. Natl. Acad. Sci. U.S.A. 98, 5116-5121.

Urlinger, S., Baron, U., Thellmann, M., Hasan, M.T., Bujard, H., and Hillen, W. (2000). Exploring the sequence space for tetracycline-dependent transcriptional activators: Novel mutations yield expanded range and sensitivity. Proc. Natl. Acad. Sci. U.S.A. 97, 7963-7968.

Wang, L., Hernandez-Alcoceba, R., Shankar, V., Zabala, M., Kochanek, S., Sangro, B., Kramer, M.G., Prieto, J., and Qian, C. (2004). Prolonged and inducible transgene expression in the liver using gutless adenovirus: A potential therapy for liver cancer. Gastroenterology 126, 278-289.

Wang, Y., O'Malley, B.W., Jr., Tsai, S.Y., and O'Malley, B.W. (1994). A regulatory system for use in gene transfer. Proc. Natl. Acad. Sci. U.S.A. 91, 8180-8184.

Xia, C., Cheshire, J.K., Patel, H., and Woo, P. (1997). Cross-talk between transcription factors NF- $\kappa \mathrm{B}$ and C/EBP in the transcriptional regulation of genes. Int. J. Biochem. Cell Biol. 29, 1525-1539.
Yang, J., Fan, G.H., Wadzinski, B.E., Sakurai, H., and Richmond, A. (2001). Protein phosphatase 2A interacts with and directly dephosphorylates RelA. J. Biol. Chem. 276, 47828-47833.

Zabala, M., Wang, L., Hernandez-Alcoceba, R., Hillen, W., Qian, C., Prieto, J., and Kramer, M.G. (2004). Optimization of the Tet-on system to regulate interleukin 12 expression in the liver for the treatment of hepatic tumors. Cancer Res. 64, 2799-2804.

Zhang, G., Budker, V., and Wolff, J.A. (1999). High levels of foreign gene expression in hepatocytes after tail vein injections of naked plasmid DNA. Hum. Gene Ther. 10, 1735-1737.

Address reprint requests to: Dr. Mercedes Reboredo Laboratorio 408

División de Hepatologia y Terapia Genica Departamento de Medicina Interna CIMA c/Pio XII $n^{\circ} 55$ 31008 Pamplona, Spain

E-mail: mmreboredo@unav.es

Received for publication April 30, 2008; accepted after revision August 21, 2008.

Published online: October 29, 2008. 
